\title{
Sleep Disorders Caused by Depression
}

\author{
Ibrahim AbduIRahman, Uzair Yaqoob* and Tariq Ali Bhatti
}

Sindh Medical College, Dow University of Health Sciences, Karachi, Pakistan

*Corresponding author: Yaqoob U, Sindh Medical College, Dow University of Health Sciences, Karachi, Sindh 74200, Pakistan, Tel: 261341420161; E-mail: ozair_91393@hotmail.com

Received date: April 22, 2018; Accepted date: April 30, 2018; Published date: May 07, 2018

Copyright: ( 2018 AbdulRahman I, et al. This is an open-access article distributed under the terms of the Creative Commons Attribution License; which permits unrestricted use; distribution; and reproduction in any medium; provided the original author and source are credited.

\begin{abstract}
Somnipathy can be categorized in to insomnia disorders, sleep-related breathing disorders, central disorders of hyper somnolence, circadian rhythm sleep-wake disorders, sleep-related movement disorders, parasomnias, and others. It can be primary or secondary, signs and symptoms of which include exorbitant daytime sleepiness, irregular breathing or increased movement during sleep and difficulty falling asleep. A few outcomes of sleep loss are obesity in adults and children, diabetes and impaired glucose tolerance, cardiovascular disease and hypertension, anxiety symptoms, depressed mood and alcohol use. Sleep disorders are frequently associated with psychiatric issues particularly depression. Studies have demonstrated that individuals with a sleeping disorder have a ten times risk of having depression in contrast to individuals who rest soundly. OSA is also linked with depression. SSRIs which increase 5-HT function increase REM sleep latency, and reduce REM sleep. Some individuals show significant improvements in depressive symptoms following a night of incomplete or complete lack of sleep, leading physicians to consider using sleep deprivation as an intervention. Antidepressants and other treatments for depression can have a beneficial effect on sleep. There is a need to evaluate for primary somnipathies, any medications being taken and underlying medical, psychiatric or substance use disorder.
\end{abstract}

Keywords: Sleep disorders; Somnipathy; Depression; insomnia of any etiology [3]. About seventy five percent of depressed Antidepressants; Obstructive sleep apnea patients have insomnia symptoms, and hypersomnia is present in about $40 \%$ of young depressed adults and $10 \%$ of older patients, with dominance in females [4]. Studies have demonstrated that individuals

\section{Introduction}

A sleep disorder, or somnipathy, is a medical disorder of sleep patterns. The recently released third edition of International Classification of Sleep Disorders (ICSD) identifies the major categories of sleep disorder. These are: Insomnia disorders, sleep-related breathing disorders, central disorders of hyper somnolence, circadian rhythm sleep-wake disorders, sleep-related movement disorders, parasomnias, and others [1].

Sleep disorder can both be primary or secondary to some medical disorder. Some of the signs and symptoms of sleep disorders include exorbitant daytime sleepiness, irregular breathing or increased movement during sleep and difficulty falling asleep. Sleep disorders stays a standout amongst the most widely recognized disregarded medical issues. Roughly 50 to 70 million Americans experience the ill effects from disorders of sleep and wakefulness, causing disturbance in doing day by day errands [2]. A few complexities of sleep loss are obesity in adults and children, diabetes and impaired glucose tolerance, cardiovascular disease and hypertension, anxiety symptoms, depressed mood and alcohol use. Laboratory tests that are performed to survey and along these lines treat sleep disorders include the polysomnogram (PSG), multiple sleep latency test (MSLT), maintenance of wakefulness test (M'WT), actigraphy, video-PSG, nocturnal penile tumescence monitoring (NPT) and electroencephalography (EEG) including 24-h ambulatory EEG.

Sleep disorders are frequently associated with depression. Insomnia, in particular, may occur in $60 \%-80 \%$ of depressed patients. Depressive symptoms are imperative hazard factors for insomnia, and depression is viewed as a vital co-morbid condition in patients with chronic with a sleeping disorder have a ten times risk of having depression in contrast to individuals who rest soundly. These all researches signify the prevention of depression in non-depressed individuals with insomnia symptoms.

Obstructive sleep apnea (OSA) is also linked with depression. In an investigation of 18,980 individuals in Europe directed by Stanford analyst Maurice Ohayon, individuals with depression were observed to be five times more inclined to experience the ill effects of OSA [5]. Fortunately treating OSA with continuous positive airway pressure (CPAP) may improve depression; a 2007 study of OSA patients who used CPAP for one year demonstrated symptoms of depression were significant and lasting [6].

The present first line treatment for depression is selective serotonergic drugs and there is much proof for the inclusion of serotonin (5-HT) in the pathogenesis of both depression and sleep disturbance. Selective serotonin reuptake inhibitors (SSRIs) which increase 5-HT function increase Rapid eye movement (REM) sleep latency, and reduce REM sleep. Despite the fact that SSRIs, selective nor-epinephrine reuptake inhibitors (SNRIs), and venlafaxine are effective and frequently used, they may intensify sleep disturbance early in treatment and may leave lingering sleep symptoms once mood is improved. However, in a study in which eszopiclone was added to fluoxetine in depressed patients there were significant beneficial effects, even in depressive symptoms other than insomnia items [7]. Some individuals show significant improvements in depressive symptoms following a night of incomplete or complete lack of sleep, leading physicians to consider using sleep deprivation as an intervention. Nonetheless, such upgrades are questionable and are reversed after a 
night of normal sleep, making lack of sleep an illogical remedial choice for depression. Some antidepressants can have a beneficial effect on sleep. These include mianserin, trazodone, nefazodone, and mirtazapine, as well as the older tricyclic antidepressants.

Cognitive behavioral therapies (CBT) are effective for short-term treatments of insomnia, as are hypnotic medications but resulted in few patients achieve complete remission with any mono treatment. A study done on 160 adults with persistent insomnia demonstrated that CBT, either alone or in combination with zolpidem, yielded significant improvements in sleep latency, time awake after sleep onset, and sleep efficiency during initial therapy [8].

\section{Conclusion}

To finish up it is obvious that there is a solid connection amongst depression and different sleep disorders. Physicians need to evaluate for primary somnipathies, any medications being taken and underlying medical, psychiatric or substance use disorder. Further researches need to be undertaken in developing more accurate and specific treatment for sleep disorders in depression.

\section{References}

1. Sateia MJ (2014) International classification of sleep disorders. Chest 146: 1387-1394.

2. Altevogt BM, Colten HR (2006) Sleep disorders and sleep deprivation: an unmet public health problem. National Academies Press.

3. Luca A, Luca M, Calandra C (2013) Sleep disorders and depression: brief review of the literature, case report, and nonpharmacologic interventions for depression. Clin Interv Aging 8: 1033-1039.

4. Nutt D, Wilson S, Paterson L (2008) Sleep disorders as core symptoms of depression. Dialogues Clin Neurosci 10: 329-336.

5. https://news.stanford.edu/news/2003/november5/depression.html

6. Schwartz DJ, Karatinos G (2007) For individuals with obstructive sleep apnea, institution of CPAP therapy is associated with an amelioration of symptoms of depression which is sustained long term. J Clin Sleep Med 3: 631-635.

7. Fava M, McCall WV, Krystal A, Wessel T, Rubens R, et al. (2006) Eszopiclone co-administered with fluoxetine in patients with insomnia coexisting with major depressive disorder. Biol Psychiatry 59: 1052-1060.

8. Beaulieu-Bonneau S, Ivers H, Guay B, Morin CM (2017) Long-term maintenance of therapeutic gains associated with cognitive-behavioral therapy for insomnia delivered alone or combined with zolpidem. Sleep 40. 